\title{
二代测序技术(NGS)结合遗传统计模型在微生物 分子生态学中的研究进展
}

\author{
梁雅静, 何晓青", 金一, 姜立波, 叶梅霞, 邬荣领 \\ 北京林业大学生物科学与技术学院, 北京 100083 \\ *联系人, E-mail: lenahe@bjfu.edu.cn
}

收稿日期：2019-06-11; 接受日期：2019-11-22; 网络版发表日期：2020-02-11

国家自然科学基金(批准号: 31971398, 31700633)、中央高校基本科研业务费专项(批准号: 2017JC05)和中国科学院科技服务网络计划(批准 号: KFJ-STS-ZDTP-036)资助

\begin{abstract}
摘要 近年来, 二代测序技术(next generation sequencing, NGS)的出现, 使得在短时间内准确获得微生物基因组数 据成为可能, NGS 已成为微生物分子生态学研究的有力工具. NGS具有非序列依赖性的优势, 能同时检测样品中 可培养或不可培养的、含量高或含量低的所有微生物. 基因组数据的获得越来越容易, 但人们对全基因组水平上 基因如何参与微生物与其他生物相互作用的了解依然十分有限。遗传统计模型则有助于实现将表型数据和基因 组数据相关联或将海量的基因组数据进行聚类分析、网络构建等, 极大地促进了全基因组水平上生物群体作用 机制的研究. 本文对NGS结合遗传统计模型在微生物分子生态学中的应用和未来前景进行了概述和展望, 探讨了 两者结合对于促进微生物种间互作机制研究的重要性, 并分析了两者结合在细菌表型可塑性机制方面的应用, 最 后阐述了其在构建微生物与植物互作基因调控网络中的作用, 旨在挖掘关键基因以增强植物的抗病能力.
\end{abstract}

关键词 NGS, 遗传统计模型, 微生物分子生态学, 微生物互作机制, 细菌表型可塑性, 微生物与植物互作

二代测序技术(next generation sequencing, NGS), 也被称为高通量或大规模并行测序，包括扩增子测序 (amplicon sequencing)、宏基因组测序(metagenomics sequencing)、转录组测序(transcriptome sequencing)、 全基因组重测序(whole genome sequencing)、从头测 序(de novo sequencing)、单细胞测序(single cell sequencing)等. NGS允许数千至数十亿个DNA片段同时 独立测序, 使得对一个物种(或细胞器)的基因组在短 期内进行细致全貌的分析成为可能. 此外, NGS还有 完美的定量功能, 样品中某种DNA被测序的次数可反
映样品中这种DNA的丰度, 这将极大地推动生物学和 医学的发展 ${ }^{[1,2]}$. 在过去的 10 年中, NGS已经从单纯的 研究工具转变为许多领域的常规应用, 包括微生物群 落结构、肠道微生物研究、病毒资源发掘、病原菌耐 药性、食品安全等领域 ${ }^{[3-8]}$. 该技术发展迅速, 质量不 断提高, 成本不断降低, 研究人员越来越多地应用NGS 来解决生物学问题.

单纯的测序技术所获得的信息依然是有限的, 结 合遗传统计模型则有助于将表型数据和基因组数据相 关联或将海量的基因组数据进行聚类分析、网络构建

引用格式: 梁雅静, 何晓青, 金一, 等. 二代测序技术(NGS)结合遗传统计模型在微生物分子生态学中的研究进展. 中国科学: 生命科学, 2020, 50: 207-214 Liang Y J, He X Q, Jin Y, et al. Progress in next generation sequencing (NGS) combined with genetic statistical model in microbial molecular ecology (in Chinese). Sci Sin Vitae, 2020, 50: 207-214, doi: 10.1360/SSV-2019-0172 
等. 深度挖掘重要的基因信息将极大地促进全基因组 水平上生物群体相关作用机制的研究. 全基因组关联 分析(genome-wide association studies, GWAS)是近年 来兴起的遗传统计模型, 在人类和动植物复杂性状遗 传研究中已取得初步成果 ${ }^{[9 \sim 14]}$. GWAS 是利用全基因 组范围内篎选出的高密度分子标记, 如SNP(single nucleotide polymorphism), 将分子标记与表型性状进行 关联, 篮选出最有可能影响性状的遗传变异位点 ${ }^{[15]}$. 系统作图(systems mapping)把表型作为一个动态过程 或系统来定位数量性状位点 (quantitative trait loci, QTLs), 具有更高的生物学内涵 ${ }^{[16,17]}$, 其打破传统基因 定位所利用的“生物是孤立存在”的假设, 构建出反映 复杂系统行为的微分方程, 能够定量分析表型之间互 作的遗传效应, 进一步估计这种互作对生物表型形成 的影响, 提升了基因定位的精度. 聚类是对转录组获 得的基因表达数据进行降维的有效方法, 通过对数以 万计的基因进行分簇, 每个聚簇的基因个数降到了几 百甚至几十个, 在模拟研究和实际数据中, 基于Skellam混合模型的方法表现出最佳的聚类性能 ${ }^{[18]}$; 同时 基因表达调控网络的重构是功能基因组中最具挑战性 的课题之一, 贝叶斯网络模型已成为构建基因调控网 络的有力工具 ${ }^{[19]}$. 本文围绕NGS并结合以上遗传统计 模型对微生物互作、表型可塑性、微生物与植物互作 等方面的应用研究进行了概述和展望.

\section{1 在微生物互作机制中的研究进展}

细菌间的互作是细菌适应生存的重要基础, 也是 组成和构建多样性微生物群落的基础. 微生物种间互 作是多样化和动态的, 并会对群落的结构和功能造成 重要影响 ${ }^{[7,20]}$. 微生物群落和生态系统生态学的重点 正在从获得观测数据转变为理解微生物互作的分子机 制, 并预测群落结构和功能的动态变化 ${ }^{[21]}$. 然而研究 微生物种间互作面临很多困难, 如传统的实验研究基 于微生物的分离与培养技术, 但是自然界中绝大多数 的微生物物种是不可培养的. 近年来, NGS的发展使 研究人员可以直接获得环境样品中微生物群落物种和 功能基因组的数据.

全基因组重测序是针对基因组序列已经获知的物 种(如大肠杆菌等), 并用NGS对不同个体的基因组序 列进行测定. 该方法不需要从头测序, 而是以已知序
列为模板，通过重新测序得到的序列与已知序列的比 对实现个体或群体差异性分析 ${ }^{[22]}$. 此方法可以找到大 量的单核苷酸多态性位点(SNPs)、插入缺失(insertion-deletion, InDel)、拷贝数变异(copy number variation, CNV)、结构变异位点(structure variation, SV)等 变异类型 ${ }^{[23,24]}$. 生物信息学软件能分析个体间基因组 的结构差异, 找到可能与研究目的相关的基因SNP位 点的分布. 近年来随着NGS的发展, 科研人员已经可 以更加快速地获得越来越多的基因数据, 同时也开始 认识到基因在物种互作中起重要的作用，但在全基因 组水平对基因如何调控微生物互作的理解依然十分有 限 ${ }^{[25,26]}$. 而遗传统计模型能帮助人们量化不同微生物 在群落内互作的类型和强度, 并将极大地促进全基因 组水平上的微生物种间互作机制的研究.

\section{1 全基因组重测序技术结合GWAS模型的研究 进展}

尽管细菌的基因组相对简单，但直到近年随着 NGS的发展, GWAS才被用来鉴定细菌重要表型的遗 传多态性 ${ }^{[27 ~ 29]}$. 对于一个成功的细菌GWAS来说, 首 先, 必须有一种可测定的表型和一组菌株的全基因组 分子标记, 其次, 需要正确选择遗传统计策略, 以确保 关联分析的准确性. 近年来关于细菌GWAS的报道也 在逐渐增加 ${ }^{[29,30]}$. He等人 ${ }^{[7]}$ 将 45 对大肠杆菌 (Escherichia coli)和金黄色葡萄球菌(Staphylococcus aureus)在 实验室条件下进行单独培养和两两混合培养, 得到生 长量表型数据. 选用E. coli str. K-12 substr. MG1655 和S. aureus subsp. aureus NCTC 8325作为参考基因组, 利用Hiseq 2500对90株菌株进行全基因组重测序, 得 到了高密度的SNP分子标记. 然后用GWAS分析了表 型数据和SNP分子标记的关系, 对得到的显著SNP位 点进行基因功能注释与分析, 定位出与两种菌株互作 所关联的显著基因, 其中包括 $r p o B, r p o C, a c r B$ 和 $r e l A$ 等, 这些基因影响细菌的细胞壁合成、RNA转录和氨 基酸缺乏时的严谨反应等过程.

\section{2 全基因组重测序技术结合系统作图模型的研 究进展}

虽然GWAS能识别细菌中高密度的分子标记, 将 细菌的表型数据和基因型相关联, 可用于分析细菌静 态表型的遗传机制 ${ }^{[7]}$, 但由于细菌互作是一个动态复 
杂的过程，传统的GWAS分析方法无法深入解析细菌 互作的遗传机制，因此还需要开发更复杂的遗传统计 模型来获悉. $\mathrm{Wu}$ 等人 ${ }^{[31]}$ 把反映生物学现象的微分方 程与基因定位相结合，用不同的方程来描述系统内不 同组成成分在自然法则下的互作，推导出系统作图的 理论框架. 系统作图的重要优势在于能够刻画动态复 杂的表型, 进而定位出影响表型以及表型系统的 QTLs，这些QTLs决定了表型系统中个体的组成和它 们之间的联系. 系统作图把表型作为一个动态过程或 系统来定位QTLs, 具有更高的生物学内涵, 是GWAS 理论的进一步扩展. 其打破传统基因解析所利用的“生 物是孤立存在”的假设, 构建出动态数学模型, 定量分 析生物之间竞争、协作等互作策略，进一步估计这种 互作对生物表型形成的影响，提升了基因解析的精度. 不仅能估计个体基因对自身表型的直接影响，还能估 计个体基因对与其共处的生物表型的间接影响，而且 还能估计出不同个体间的基因通过信号传导对整个生 物表型的互作影响, 使QTLs定位更具生物学内涵. 目 前，在系统作图框架下已集成描述生物钟的微分方程 和刻画病毒动态变化的微分方程等 ${ }^{[32,33]}$. 最近, 博弯 论被引入该理论框架, 用于解析共培养细菌之间相互 竞争与合作的方式与强度，基于此，研究人员提出了 竞争合作定位模型 ${ }^{[17]}$.Jiang等人 ${ }^{[17]}$ 发现，两个不同细 菌在共培养条件下的生长, 与同一细菌在单独培养条 件下的生长存在明显差异, 而这种差异就是表型可塑 性并受基因控制。进一步利用博弯论解析共培养中两 个细菌间相互竞争与合作的方式与强度后发现，混合 培养中细菌的表型性状不仅受细菌自身的基因所影响 (直接作用, direct effect), 还受互作细菌基因的影响(间 接作用, indirect effect), 以及来自不同细菌的基因之间 交互作用的影响. 研究人员提出“基因组-基因组上位 互作”(genome-genome epistasis)概念，来描述这些复 杂的基因调控关系. 研究发现，被传统模型所忽略的 间接作用与基因组交互作用，能解释几乎 $1 / 2$ 的遗传力 (heritability). 这一重要发现为复杂性状遗传机理的研 究提供了新的观点.

\section{2 在细菌表型可塑性和适应性进化中的研 究进展}

表型可塑性是基因型与环境互作的结果, 被定义
为个体在响应环境变化时改变表型的能力, 较强的表 型可塑性能够有效地应对自然选择或其他胁迫因 素 ${ }^{[34]}$. 表型可塑性变异在不同物种中广泛存在, 固定 基因型的生物体通过复杂的调控系统, 产生不同的表 型能够更有效地适应新环境, 推动物种进化. 环境发 生剧烈变化, 例如目前面临的全球气候变化, 会迫使 各类生物体尽快适应新环境, 避免物种灭绝 ${ }^{[35]}$. 在特 定个体与环境互作中, 表型的变化同时受到基因变异 和表型可塑性的影响, 较强的表型可塑性能够有效地 应对自然选择或其他胁迫因素. 过去的十多年中, 大 量报道表明, 表型可塑性从不同角度在多种物种的适 应性进化中起到了关键性作用，包括多物种共存 ${ }^{[36]}$ 、 逃避物种灭绝 ${ }^{[37]}$ 及影响交配行为 ${ }^{[38]}$ 等. 表型可塑性已 逐渐成为生态学、发育生物学、进化生物学等领域的 研究热点.

目前表型可塑性的研究工作在各种多细胞生物 (包括昆虫、爬行动物、植物及人类等)中积累了大量 成果 ${ }^{[38-40]}$, 而对微生物表型可塑性基因的研究还较为 欠缺. 微生物的表型包括很多方面, 如个体生长、群 体生长、基因表达及耐药性等. 在不同环境因素的影 响下, 为了确保物种存活, 特定基因型的微生物需要 具备对不同胁迫的快速响应能力, 表型可塑性基因在 这一过程中起重要作用. 虽然微生物在生态环境中扮 演着重要角色, 但对这些单细胞生物表型可塑性的研 究还比较有限. 对单细胞真核生物酿酒酵母(Saccharomyces cerevisiae) 在不同环境胁迫下基因的转录变化分 析发现, 转录可塑性在适应环境变化的过程中起决定 性作用 ${ }^{[41]}$. 对海洋微生物的研究发现, 表型可塑性影 响微生物群落功能变化 ${ }^{[18]}$, 较强的表型可塑性加强了 微生物的环境适应能力 ${ }^{[42]}$. 有研究者从极地海洋、南 极低盐和高盐度南极湖 3 个不同环境分离培养了 11 株 极地甲藻, 测定其在盐度胁迫时的表型可塑性, 发现与 海洋祖先菌株相比, 湖泊菌株的表型可塑性较高, 这可 能是由于湖泊系统中盐度大幅度变化造成的 ${ }^{[42]}$. 在细 菌的研究工作中发现, 表型可塑性不仅能够有效地响 应环境变化 ${ }^{[43]}$, 还能够影响大肠杆菌细胞分裂的对称 选择 ${ }^{[44]}$ 及霍乱弧菌(Vibrio cholerae)生物膜的形成 ${ }^{[45]}$.

同一基因型的菌株在不同环境中所表现出的可塑 性程度存在差异, 虽然可塑性不能影响个体的基因型, 但不同基因型个体对同一环境变化产生的不同可塑性 将影响适应性进化. 如果菌株的可塑性能够帮助其在 
不利环境中生存, 相应表型的改变则会被强化. 在微生 物与环境互作的过程中, 基因变异产生的表型改变往 往更容易被保存下来，但基因变异是随机的，只有与 适应性进化方向一致的才能遗传下去. 表型可塑性则 在一定程度上可以抵消环境变化对微生物生长成的影 响, 使其表现出更好的生存优势. 当细菌面临一定浓度 抗生素的选择压力时, 其群体生长会受到极大的抑制, 菌株必须保持一定生长能力才有可能实现适应性进 化. 可以想象菌株的生长可塑性基因在应对抗生素环 境时将起重要作用, 具有较强生长可塑性的个体很可 能表现出更好的生存优势, 发展出耐药菌的可能性也 更大 ${ }^{[46]}$.

传统的可塑性研究往往停留在表型层面, 或仅关 注特定基因, 忽略了基因组中其他因素对可塑性的影 响. 随着NGS和细菌GWAS的发展, 利用遗传统计模型 研究微生物表型可塑性为解析细菌表型遗传机制提供 了一种新的思路. 在表型可塑性研究中, 来源于不同环 境的表型数据很难用传统单变量GWAS进行分析. 将 多元线性混合模型算法加入GWAS能够实现多变量表 型分析 ${ }^{[15]}$, 同时将多组表型数据与遗传标记相关联, 更适合表型可塑性数据的分析. 在众多GWAS分析软 件中, GEMMA(genome-wide efficient mixed model association)可以将标准线性混合模型扩展到多变量表 型线性混合模型, 且同时考虑种群结构及亲缘关系 ${ }^{[47]}$. Rong等人 ${ }^{[46]}$ 借助GEMMA软件的双变量静态GWAS对 金黄色葡萄球菌在不同浓度万古霉素培养基中的生长 可塑性进行了分析, 共定位到227个显著SNPs. 其中 14 个SNPs位于功能已被注释的基因上，这些基因大多与 细菌的生长代谢及抗逆性有关. 根据 $p$ 值 ( $p$ value)大小 和出现频率等发现, 4个显著SNPs很可能在金黄色葡 萄球菌生长可塑性调控中起重要作用. 对表型可塑性 基因的深入研究, 能够进一步揭示微生物基因与环境 交互作用的机制, 为解析微生物适应性进化提供重要 资料.

\section{3 在植物-病原体互作基因网络构建中的研 究进展}

当前植物病原体互作越来越成为热点问题, 随着 转录组技术的发展, 研究者在组学水平对植物-病原体 互作机制的了解越来越多. 通过转录组方法人们能够
发现植物被病原体侵染后的基因表达变化, 以及在病 原菌侵入植物后病原菌的基因表达变化. 单纯通过测 序技术获得的信息有限, 但遗传统计模型的发展结合 转录组测序技术为植物病原体互作打开了一扇新的大 门, 通过建立遗传统计模型, 可以将植物和病原体的基 因按照不同的表达模式进行聚类, 从而更好地研究植 物-病原体互作.

聚类分析作为一种强有力的分析工具在基因表达 数据的分析中有被广泛应用, 虽然有很多方法可以完 成基因表达数据的聚类, 但基于模型的聚类方法有较 好的统计理论支撑、成熟的模型选择方法等优势, 是 基因表达数据分析的首要选择. 针对转录组动态基因 表达数据, 基于泊松和负二项模型的方法能够对其进 行聚类分析, 然而这些方法只能对生物响应单一环境 下的基因表达模式进行聚类分析，如果生物从适宜的 环境突然转换到逆境环境, 其基因表达可产生可塑性 响应 ${ }^{[48]}$, 只有对产生可塑性响应基因的表达模式进行 聚类才能更精确地挖掘生物响应逆境的关键基因. 两 个环境下基因表达的差值是量化基因表达可塑性响应 的重要方式 ${ }^{[49]}$, 对基因动态表达的可塑性响应进行聚 类解析, 更有助于后续的基因调控网络构建, 为最终 关键功能基因的篮选奠定基础.

\section{1 转录组测序技术结合Skellam混合模型的研究 进展}

Skellam分布是一个描述两个独立随机变量差值 的离散概率分布, 两个变量都服从泊松分布. Jiang等 人 $^{[50]}$ 基于Skellam分布, 构建了Skellam混合模型联合 似然, 并推导EM算法对未知参数进行估计, 同时提出 两个假设检验对聚类内基因表达模式以及不同聚类间 的表达模式是否存在差异进行检测. 通过模拟研究, 他 们比较了不同的聚类中心初值选取方法、不同的数据 标准化方法对聚类性能的影响, 同时也对新方法的聚 类数量判定以及表达模式估计的准确度进行了探究, 发现该方法能够对转录组动态基因的可塑性表达进行 聚类分析. Skellam混合模型表现出的最优聚类性能, 明显高于 $\mathrm{k}$ 均值聚类算法( $\mathrm{k}$-means clustering algorithm)和SOM算法(self-organizing maps) ${ }^{[50]}$.

孔维文 ${ }^{[51]}$ 对灰葡萄狍菌(Botrytis cinerea) 侵染黄 瓜(Cucumis sativis) 前后的样本进行了转录组测序, 得 到了238341648个高质量的序列, 并通过差异表达基因 
分析, 获得了 4021 个黄瓜差异基因和671 个灰葡萄孢菌 差异基因. 为了更加深入地挖掘植物-病原体互作的机 制，研究人员用Skellam混合模型，将差异基因进行聚 类分组, 黄瓜差异基因聚类到17组, 灰葡萄孢菌差异基 因聚类到 12 组, 降低了数据维度. 利用 $\mathrm{GO}$ 富集分析与 数据库的注释对聚类结果进行了进一步挖掘, 研究了 哪些基因可能参与了二者的互作，从而分析基因的可 塑性表达模式，进一步解析了灰葡萄孢菌侵染黄瓜后 的内在调控机制.

\section{2 贝叶斯网络在基因调控网络构建中的研究 进展}

重构基因调控网络有助于了解生物过程的关键问 题, 随着测序技术的发展, 产生的海量基因表达数据与 一定计算方法的结合可以重构基因调控网络. 研究基 因调控网络的目的在于利用基因表达数据，重现基因 互作的拓扑结构，达到揭示基因复杂的作用机理及基 因功能信息的目的 ${ }^{[19]}$. 目前, 已有许多模型被应用于 基因调控网络的构建, 其中贝叶斯网络(Bayesian networks)模型以其坚实的理论基础、表述自然的知识结 构、灵活的推理能力以及高效的决策机制使其应用范 围越来越广泛，已成为构建基因调控网络的一种有力 工具 ${ }^{[52]}$.

Zhang等人利用贝叶斯网络对上述灰葡萄孢菌侵 染黄瓜的聚类结果进行基因网络构建，以进一步挖掘 植物-病原体互作的关键基因(hub genes)(未出版, BMC genomics修改中). 在黄瓜的差异基因聚类分组 中, 第 12 组中有 543 个基因, 经过贝叶斯网络构建后, 3 个基因被标注为 $\mathrm{hub}$ g e nes, 其中编号为 Csa2G07544029的基因被注释为表达抗病蛋白RPS2 (disease resistance protein RPS2), 富集到“植物-病原体 互作”(plant-pathogen interaction)的KEGG通路中, 表明 Csa2G07544029在黄瓜抵御灰葡萄孢菌侵染的过程中 起非常重要的作用. 同样, 在灰葡萄孢菌差异基因聚类 分组中，第10组有 15 个基因，其中基因B0510_3699在 贝叶斯网络构建中被标注为hub gene, 该基因编码 $1,4-$ $\beta-\mathrm{D}$-葡聚糖纤维二糖水解酶. GO富集分析显示, 该酶 参与调节水解酶的活性或水解O-糖基化合物 (hydrolase activity or hydrolyzing O-glycosyl compounds, GO: 0004553)，表明此酶在病原体降解植物细胞壁或利用 植物细胞壁多糖时扮演重要角色.

\section{4 展望}

NGS技术的发展使得基于非培养方法研究微生物 成为可能, 并推动微生物研究进入黄金发展时期. 近 10 年来, NGS因其快速和灵敏的检测特点成为微生物分 子生态学研究的强有力工具. 遗传统计模型以生物学 数据为基础, 结合数学、统计学、计算机科学等方法 被用来进行分子遗传学等方面的研究. 两者相结合已 应用在微生物种间互作、微生物与环境互作, 以及微 生物与植物互作等研究中, 并取得了非常好的效果. 随着对气候变化、微生物耐药性、肠道微生物等方面 研究的深入, 两种方法的融合将在微生物生态学分子 机理研究上取得更多的成果.

第三代测序技术以Pacific BioSciences (PacBio)和 Oxford Nanopore Technologies (ONT)等为代表, 实现 了单分子水平上的基因测序，虽然受到测序错误率高 和配套软件缺乏的困扰，但在读长、测序速度等方面 的优势正在逐渐突显 ${ }^{[53,54]}$. 随着第三代测序技术的错 误率降低和计算效率提高, 结合遗传统计模型, 将进 一步推动微生物分子生态学的相关研究.

\section{参考文献}

1 Gu W, Miller S, Chiu C Y. Clinical metagenomic next-generation sequencing for pathogen detection. Annu Rev Pathol Mech Dis, 2019, 14: 319338

2 Sun J Y, Yang C, Hu X W, et al. A cost-efficient method for sequencing the human whole mitochondrial genome utilizing multiplex PCR-based next generation sequencing (in Chinese). Sci Sin Vitae, 2017, 47: 396-402 [孙嘉仪, 杨超, 胡晓雯, 等. 一种基于多重PCR的人类线粒体基因组 高通量测序方法. 中国科学: 生命科学, 2017, 47: 396-402]

3 Quainoo S, Coolen J P M, van Hijum S A F T, et al. Whole-genome sequencing of bacterial pathogens: The future of nosocomial outbreak analysis. Clin Microbiol Rev, 2017, 30: 1015-1063

4 Jagadeesan B, Gerner-Smidt P, Allard M W, et al. The use of next generation sequencing for improving food safety: Translation into practice. 
Food Microbiol, 2019, 79: 96-115

5 Conrads G, Abdelbary M M H. Challenges of next-generation sequencing targeting anaerobes. Anaerobe, 2019, 58: 47-52

6 Perruzza L, Gargari G, Proietti M, et al. T follicular helper cells promote a beneficial gut ecosystem for host metabolic homeostasis by sensing microbiota-derived extracellular ATP. Cell Rep, 2017, 18: 2566-2575

$7 \mathrm{He} \mathrm{X}$, Jin Y, Ye M, et al. Bacterial genetic architecture of ecological interactions in co-culture by GWAS-taking Escherichia coli and Staphylococcus aureus as an example. Front Microbiol, 2017, 8: 2332

8 Qian Y J, Xu Y, Zhou Q, et al. Application of next-generation sequencing technology for plant virus identification (in Chinese). Sci Sin Vitae, 2014, 44: 351-363 [钱亚娟, 徐毅, 周琦, 等. 利用深度测序技术发掘植物病毒资源. 中国科学: 生命科学, 2014, 44: 351-363]

$9 \mathrm{Xu}$ P, Zhang Z, Zhang F, et al. Identification of candidate genes for hematological traits by integrating gene expression profiling and genomewide association study in a porcine model (in Chinese). Sci Agri Sin, 2016, 49: 348-360 [徐盼, 张震, 章峰, 等. 整合数字基因表达谱与全基因 组关联分析鉴定猪血液性状候选基因. 中国农业科学, 2016, 49: 348-360]

10 Wu X P. GWAS and genomic prediction based on markers of SNP-chips and sequence data in dairy cattle populations (in Chinese). Dissertation for Doctoral Degree. Beijing: China Agricultural University, 2014 [吴晓平. 基于SNP芯片和全测序数据的奶牛全基因组关联分析和基因组选 择研究. 博士学位论文. 北京: 中国农业大学, 2014]

11 Duan W W, Zhao Y, Zhang L W, et al. Strategies of genetic risk prediction with lung cancer GWAS data (in Chinese). Chin J Health Stat, 2015, 32: 554-557 [段巍巍, 赵杨, 张丽伟, 等. 使用肺癌GWAS数据进行遗传风险预测的方法和策略研究. 中国卫生统计, 2015, 32: 554-557]

12 Zheng H F, Forgetta V, Hsu Y H, et al. Whole-genome sequencing identifies EN1 as a determinant of bone density and fracture. Nature, 2015, 526: $112-117$

13 Shungin D, Winkler T W, Croteau-Chonka D C, et al. New genetic loci link adipose and insulin biology to body fat distribution. Nature, 2015, 518: $187-196$

14 Dhindsa R S, Goldstein D B. From genetics to physiology at last. Nature, 2016, 530: 162-163

15 Chen P E, Shapiro B J. The advent of genome-wide association studies for bacteria. Curr Opin Microbiol, 2015, 25: 17-24

16 Zhu X, Jiang L, Ye M, et al. Integrating evolutionary game theory into mechanistic genotype-phenotype mapping. Trends Genets, 2016, 32: 256268

17 Jiang L, He X, Jin Y, et al. A mapping framework of competition-cooperation QTLs that drive community dynamics. Nat Commun, 2018, 9: 3010

18 Beier S, Rivers A R, Moran M A, et al. Phenotypic plasticity in heterotrophic marine microbial communities in continuous cultures. ISME J, 2015, 9: 1141-1151

19 Ge L L. The research of constructing gene regulatory network based on model of dynamic Bayesian (in Chinese). Dissertation for Master's Degree. Hefei: Hefei University of Technology, 2010 [葛玲玲. 基于动态贝叶斯模型的基因调控网络构建方法研究. 硕士学位论文. 合肥: 合 肥工业大学, 2010]

20 Faust K, Raes J. Microbial interactions: From networks to models. Nat Rev Microbiol, 2012, 10: 538-550

21 He W Q, Zhang Q G. Advances in microbial interaction research: From observation to prediction (in Chinese). J Beijing Norm Univ (Nat Sci), 2016, 52: 809-815 [郝祎祺, 张全国. 微生物相互作用研究进展: 从观察到预测. 北京师范大学学报(自然科学版), 2016, 52: 809-815]

22 Ley T J, Mardis E R, Ding L, et al. DNA sequencing of a cytogenetically normal acute myeloid leukaemia genome. Nature, 2008, 456: 66-72

23 Liti G, Carter D M, Moses A M, et al. Population genomics of domestic and wild yeasts. Nature, 2009, 458: 337-341

24 Irvine D V, Goto D B, Vaughn M W, et al. Mapping epigenetic mutations in fission yeast using whole-genome next-generation sequencing. Genome Res, 2009, 19: 1077-1083

25 Zhang Z R, Li J T, Liang Y J, et al. Analysis of interaction mode between Escherichia coli and Staphylococcus aureus by systems mapping (in Chinese). Microbiol China, 2019, 46: 292-300 [张佐然, 李金婷, 梁雅静, 等. 利用系统作图(systems mapping)研究大肠埃希菌和金黄色葡萄 球菌的互作遗传机制. 微生物通报, 2019, 46: 292-300]

26 Whitham T G, Bailey J K, Schweitzer J A, et al. A framework for community and ecosystem genetics: From genes to ecosystems. Nat Rev Genet, 2006, 7: 510-523

27 Read T D, Massey R C. Characterizing the genetic basis of bacterial phenotypes using genome-wide association studies: A new direction for bacteriology. Genome Med, 2014, 6: 109

28 Yang C, Yang R F, Cui Y J. Bacterial genome-wide association study: Methodologies and applications (in Chinese). Hereditas, 2018, 40: 57-65 
[杨超, 杨瑞馥, 崔玉军. 细菌全基因组关联研究的方法与应用. 遗传, 2018, 40: 57-65]

29 Chen N, Zhu J, Ye M X, et al. Studies the evolutionary mechanism of Escherichia coli and Staphylococcus aureus by GWAS (in Chinese). Acta Microbiol Sin, 2017, 57: 526-538 [陈南, 朱璟, 叶梅霞, 等. GWAS研究大肠杆菌和金黄色葡萄球菌种间互作进化机制. 微生物学报, 2017, 57: 526-538]

30 Power R A, Parkhill J, de Oliveira T. Microbial genome-wide association studies: Lessons from human GWAS. Nat Rev Genet, 2016, 18: 41-50

31 Wu R, Cao J, Huang Z, et al. Systems mapping: How to improve the genetic mapping of complex traits through design principles of biological systems. BMC Syst Biol, 2011, 5: 84

32 Luo J, Hager W W, Wu R. A differential equation model for functional mapping of a virus-cell dynamic system. J Math Biol, 2010, 61: 1-15

33 Fu G, Luo J, Berg A, et al. A dynamic model for functional mapping of biological rhythms. J Biol Dyn, 2011, 5: 84-101

34 Pigliucci M, Murren C J, Schlichting C D. Phenotypic plasticity and evolution by genetic assimilation. J Exp Biol, 2006, 209: 2362-2367

35 Nadeau C P, Urban M C, Bridle J R. Climates past, present, and yet-to-come shape climate change vulnerabilities. Trends Ecol Evol, 2017, 32 : $786-800$

36 Turcotte M M, Levine J M. Phenotypic plasticity and species coexistence. Trends Ecol Evol, 2016, 31: 803-813

37 Chevin L M, Lande R, Mace G M. Adaptation, plasticity, and extinction in a changing environment: Towards a predictive theory. PLoS Biol, 2010, 8: e1000357

38 Filice D C S, Long T A F. Phenotypic plasticity in female mate choice behavior is mediated by an interaction of direct and indirect genetic effects in Drosophila melanogaster. Ecol Evol, 2017, 7: 3542-3551

39 Dicke M. Plant phenotypic plasticity in the phytobiome: A volatile issue. Curr Opin Plant Biol, 2016, 32: 17-23

40 Emmons M F, Faião-Flores F, Smalley K S M. The role of phenotypic plasticity in the escape of cancer cells from targeted therapy. Biochem Pharmacol, 2016, 122: 1-9

41 Mattenberger F, Sabater-Muñoz B, Toft C, et al. The phenotypic plasticity of duplicated genes in Saccharomyces cerevisiae and the origin of adaptations. G3 (Bethesda), 2017, 7: 63-75

42 Rengefors K, Logares R, Laybourn-Parry J, et al. Evidence of concurrent local adaptation and high phenotypic plasticity in a polar microeukaryote. Environ Microbiol, 2015, 17: 1510-1519

43 Kümmerli R, Jiricny N, Clarke L S, et al. Phenotypic plasticity of a cooperative behaviour in bacteria. J Evol Biol, 2009, 22: 589-598

44 Lele U N, Baig U I, Watve M G. Phenotypic plasticity and effects of selection on cell division symmetry in Escherichia coli. PLoS ONE, 2011, 6: e14516

45 Yan J, Nadell C D, Bassler B L. Environmental fluctuation governs selection for plasticity in biofilm production. ISME J, 2017, 11: 1569-1577

46 Rong M, Zheng X, Ye M, et al. Phenotypic plasticity of Staphylococcus aureus in liquid medium containing vancomycin. Front Microbiol, 2019, 10: 809

47 Zhou X, Stephens M. Efficient multivariate linear mixed model algorithms for genome-wide association studies. Nat Methods, 2014, 11: 407-409

48 Pfenning A R, Schwartz R, Barth A L. A comparative genomics approach to identifying the plasticity transcriptome. BMC Neurosci, 2007, 8: 20

49 Hoffmann A A, Willi Y. Detecting genetic responses to environmental change. Nat Rev Genet, 2008, 9: 421-432

50 Jiang L, Mao K, Wu R. A skellam model to identify differential patterns of gene expression induced by environmental signals. BMC Genomics, 2014, 15: 772

51 Kong W W. Cucumber gray mold PMA-qPCR method and large scale transcriptome analysis during infection (in Chinese). Dissertation for Master's Degree. Beijing: Beijing Forestry University, 2016 [孔维文. 黄瓜灰霉病PMA-qPCR分子诊断及互作转录组学研究. 硕士学位论文. 北京: 北京林业大学, 2016]

52 Liu P. Construction of gene regulatory networks based on Bayesian approaches (in Chinese). Dissertation for Master's Degree. Suzhou: Soochow University, 2013 [刘攀. 基于贝叶斯方法的基因调控网络构建. 硕士学位论文. 苏州: 苏州大学, 2013]

53 Xiao C L, Chen Y, Xie S Q, et al. Mecat: Fast mapping, error correction, and de novo assembly for single-molecule sequencing reads. Nat Methods, 2017, 14: 1072-1074

54 Liu Y X, Qin Y, Guo X X, te al. Methods and applications for microbiome data analysis (in Chinese). Hereditas, 2019, 49: 845-862 [刘永金金, 秦 媛, 郭晓璇, 等. 微生物组数据分析方法与应用. 遗传, 2019, 49: 845-862] 


\title{
Progress in next generation sequencing (NGS) combined with genetic statistical model in microbial molecular ecology
}

\author{
LIANG YaJing, HE XiaoQing, JIN Yi, JIANG LiBo, YE MeiXia \& WU RongLing \\ College of Biological Sciences and Technology, Beijing Forestry University, Beijing 100083, China
}

\begin{abstract}
In recent years, the emergence of next generation sequencing (NGS) makes it possible to obtain the data of microbial genome accurately in a short period of time, and it has become a powerful tool for microbial molecular ecology research. NGS has the advantage of non-sequence dependence and it can simultaneously detect nearly all culturable and non-culturable microorganisms which have high or low contents. With the explosion of sequencing data generated by NGS and multi-omics techniques, there is a growing need for the powerful genetic statistical models to generate meaningful hypotheses about the mechanisms underlying biological interaction. In this paper, we mainly discuss the application and prospect of NGS combined with the genetic statistical models in microbial molecular ecology. The combination of the two in promoting the study of microbial interspecific interaction is discussed. Then we analyze the application of the combination in the study of bacterial phenotypic plasticity. Finally, we describe its application in constructing gene regulatory network of microbe-plant interaction, which aims to identify key genes to enhance the disease resistance of plants.
\end{abstract}

NGS, statistical model, microbial molecular ecology, mechanisms of microbial interactions, bacterial phenotypic plasticity, microbial-plant interactions

doi: $10.1360 / \mathrm{SSV}-2019-0172$ 